\title{
Validating simulations of development outcomes in the Mekong region
}

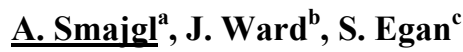 \\ ${ }^{a}$ CSIRO Ecosystem Sciences, Townsville 4810 Queensland, Australia \\ ${ }^{b}$ CSIRO Ecosystem Sciences, Dutton Park 4102 Queensland, Australia \\ ${ }^{c}$ CSIRO Ecosystem Sciences, Highett 3190 Victoria \\ Email: alex.smajgl@,csiro.au
}

\begin{abstract}
The wider Mekong region is experiencing an unprecedented influx of private and foreign investment, which is transforming many areas of the six riparian countries. Most investments target access to natural resources, demoting the outcomes of development objectives such as poverty alleviation to coincidental side-effects. The Mekong region simulation (Mersim) model aims to improve the understanding of unintended side-effects, by providing the computational foundation for a participatory learning process involving decision makers and decision influencers. The model simulates the poverty and economic outcomes of scenarios selected and designed by participating decision makers, including the impact of Mekong mainstream dams, the impact of payments for ecosystem services on land use change, the impact of large scale irrigation, and the impact of sealevel rise. The complexity of these social-ecological processes emphasises the relevance of iterative participant validation. This paper describes a process that involves a pattern-based validation technique as an alternative to a numerical validation method. The patterns were introduced in structured workshops to challenge causal beliefs elicited during the participatory process and validated through facilitated stakeholder discussions. This paper explains how, as an initial response, stakeholders actively defend currently held beliefs. When confronted with the counterintuitive results and outcomes simulated by the Mersim model, the discussion shifted to understanding model mechanisms, allowing stakeholders to explore alterative beliefs and some held beliefs were readily amended, validating some important results of the Mersim model.
\end{abstract}

Keywords: $\quad$ Agent-based modelling; Validation; Mekong. 


\section{INTRODUCTION}

The past thirty years have witnessed a substantial transformation of scientific methodology towards transdisciplinary approaches. Largely driven by sustainability related policy concerns substantial efforts have been devoted to appraising and developing methods that can function as transdisciplinary platforms (Smajgl and Ward, in review-b). Agent-based modelling emerged from computer science and is now being increasingly endorsed and utilised across many disciplines. A critical advantage is the strength to model explicitly disaggregated human decision-making processes as part of highly complex, spatial interactions of ecological and social systems (Gilbert, 2008; Smajgl et al., 2009a; Smajgl et al., 2008; Troitzsch, 2013). A rapidly increasing number of applications have developed complex models that allow for the observation of emerging phenomena to inform decision making processes (Matthews et al., 2007; Sawyer, 2005; Smajgl and Bohensky, 2013; Smajgl et al., 2009b). The increasing complexity of large-scale models that often incorporate many stochastic parameters (Smajgl, 2010; Young, 2006) diminishes the defensibility of point predictions and attendant numerical confirmation. It is no longer imperative to validate a model in the traditional sense where one analyses the ability of the model to accurately make point predictions. Rather, the application focus has shifted to providing a model that can be effective in a participatory process to facilitate learning or conflict resolution (Barreteau et al., 2003; Bousquet et al., 1999). This shift has consequences for model validation, a critical modelling step.

The term validation is commonly used to describe the process of comparing simulation results with the (empirical or theoretical) target system. In addition to such an output-focused perspective the conceptualisation and data that feeds into model implementation can be validated. Actions concerned with the computational implementation of a model design and the correctness code is normally referred to as verification. David (2013), Windrum et al. (2007), Moss (2008) and Amblard et al. (2007) provide excellent overviews to the field of validation of agent-based models. Validation can involve various different techniques, as described in David (2013) and depending on the research goal the set of techniques might change. For instance, for predictive purposes the comparison with historical data or particular events (historical validity or event validity) by retrodiction or backcasting become important validation techniques. For the purpose of this paper we introduce an explicit distinction between the validation of numerical values and patterns similar to Alcamo (2001), Moss and Edmonds (2005), Moss (2008) and Smajgl et al. (2011b).

The paper discusses validation in the empirical context of the Mekong region. For this purpose more contextual background is provided in the next section before specifying details of the Mekong region (Mersim) model, its results and implemented validation techniques.

\section{THE MEKONG REGION DEVELOPMENT CONTEXT}

The wider Mekong Region comprises of Vietnam, Cambodia, Lao PDR, Thailand and China's Province Yunnan. This region experiences an unprecedented influx of private and public investment, largely establishing hydropower, rubber plantations, irrigation schemes for food and energy crops, and mining (Smajgl and Ward, 2013). The majority of current investments demotes development objectives such as poverty alleviation to a coincidental side-effect as primary performance criteria are focused on expanding profit margins, securing access to natural resources, and safe-guarding sovereign energy and food security. Understanding unintended side-effects of investments in the Mekong region is critical if decision makers aim to achieve poverty alleviation targets. An effective understanding can allow for selecting and amending investments to safeguard or improve the livelihoods of the poor. Due to the complexity of this task and the relevance of human behaviours, expressed as household responses to potential changes in their social, economic and bio-physical environment, agent-based modelling was selected as the approach most likely to generate cogent insights for decision makers. The model was developed and deployed during a participatory learning process (Foran et al., in print; Smajgl et al., in review; Smajgl and Ward, in review-a, b).

\section{THE MEKONG REGION SIMULATION (MERSIM) MODEL}

The development of an agent-based model for the wider Mekong region focused on simulating household responses to a set of environmental changes, including

- the availability of irrigation in northeast Thailand and in the Nam Ngum catchment in Lao PDR,

- salinity intrusion and changes to flooding in Vietnam's Mekong Delta,

- Payments for ecosystem services (PES) to manage the expansion of rubber plantations in Xishuangbanna, Yunnan, and 
- $\quad$ the impact of hydropower in Lao PDR on livelihoods around the Tonle Sap, Cambodia.

The description of the agent-based model Mersim (Mekong region simulation) (Smajgl et al., 2013) follows the ODD (Overview - Design concepts - Details) protocol (Grimm et al., 2006; Grimm et al., 2010).

Purpose: The simulation model aims to facilitate parts of a larger participatory learning process (Smajgl and Ward, in review-a, b). While the overarching process allows stakeholders with competing goals to interact, the model aims to provide insights in more complex social-ecological interaction.

State variables: Household income, Household livelihoods, Household location, land cover, rice production, cassava production, rubber production, fish catch, and poverty rate.

Emergence: Emergent phenomena include poverty rate and spatial poverty patterns. Livelihood changes emerge in response to changes in environmental, economic and political factors. Land use patterns evolve as a consequence of social-environmental interaction; Fig. 1 depicts the principle model process.

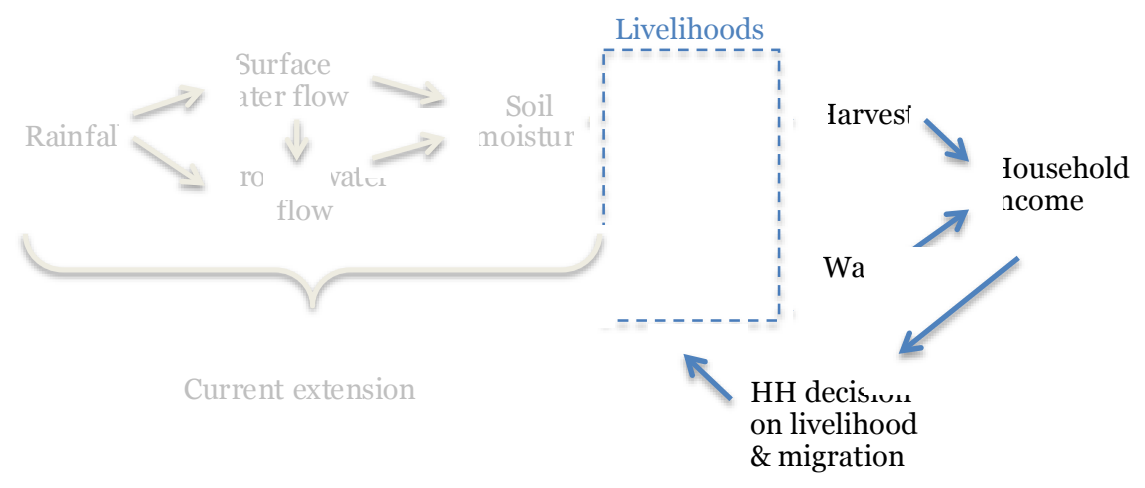

Fig. 1. Process overview of the Mekong Region model and its current extension (in grey)

Adaptation \& Objective: Household agents respond to income levels that result from paid labour and agricultural activities. Households' objectives are implicit to their behavioural response functions (or rules). Agents respond to changes based on intentional data elicited in large-scale surveys as the next Section explains in detail. No additional optimisation or satisficing assumption is implemented. Consequentially, household expectations and learning are not explicitly represented but implicitly captured by the empirically derived response strategies.

Stochasticity: Most model parameters were assumed to be stochastic, to resemble more realistic analogues of real world attributes such as crop prices, productivity, wages, and rainfall.

Initialisation: The Mersim model utilises five sets of GIS data: (1) administrative boundaries down to administrative villages, (2) soil data, (3) land cover data, (4) rainfall projections, and (5) a digital elevation model. These datasets were used to specify the artificial landscape while household survey data provided the necessary data on household attributes and behavioural responses.

The parameterisation process is described based on the framework provided in Smajgl et al. (2011a).

Fig. 2 shows the principle parameterisation steps an empirical model requires. The Mersim model formulation is based on theory conceptualising social-ecological complexity. Key dimensions of these underpinnings are the bottom-up design of a systems view that includes non-linear dynamics to interact.

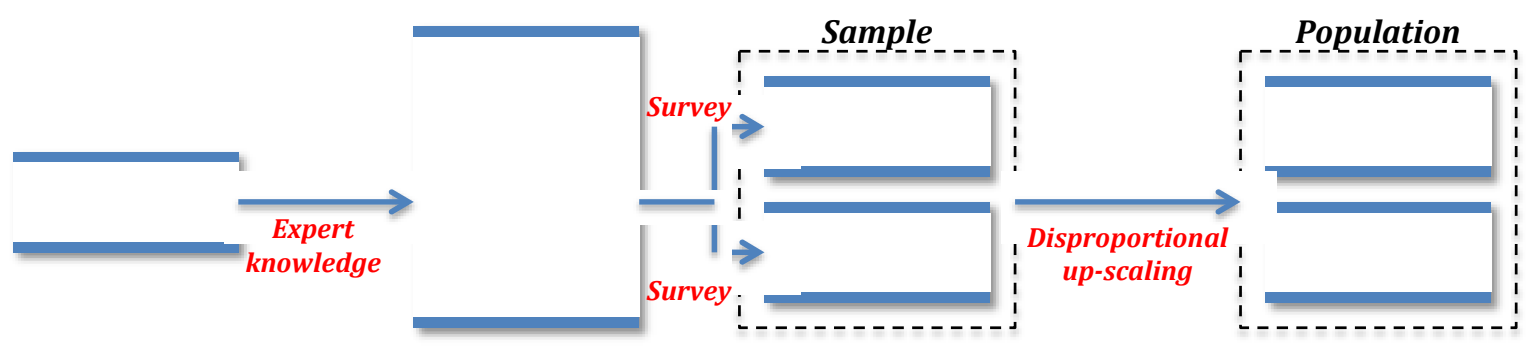

Fig. 2. Parameterisation Sequence for the Mersim model, adapted from Smajgl et al. (2011a) 
Experts helped identify principal agent classes and priority attributes, such as household agents, government agents and spatial agents. This expert-based process also identified principal agent behaviour such as the harvest of tea and the tapping of rubber. These livelihood-related activities were put into a typical seasonal production calendar and linked to specific regions and altitudes where necessary.

The next step involved the specification of household attributes and household behaviours. 5,000 randomly sampled households across the five local case study areas were surveyed to elicit key characteristics (i.e. location, household composition and size, livelihoods, production, and income), subjective wellbeing, human values, and their adaptive intentions. Intentions represent responses to questions that frame a hypothetical change. In this case the change households were asked to imagine the large-scale changes listed in section 3 and additional social and economic changes, including the substantial loss of income, emigration of other community members and climate change. Households had four principle response options

- To maintain their livelihood where they live

- To change their livelihood where they live

- To migrate out but maintain their livelihood

- To migrate out and change their livelihood

In each of these categories follow-on questions elicited adaptive impediments and specified the magnitude or type of livelihood response and/or the location for migration.

The sample data for attributes and behavioural rules was then proportionally up-scaled. The spatial distribution during the initialisation process is based on land use data. This GIS-based adjustment aims for a more realistic spatial distribution of simulated household behaviour.

Submodels: Household income is calculated in weekly time steps as the sum of all livelihoods all household members engage in. This includes the monetisation of subsidence production to avoid a misleading quantification of poverty. Poverty is calculated as the percentage of people within a (administrative) village below the official poverty line. Further hydrological submodels are currently added to link crop growth to soil moisture changes instead of harvest data elicited by the household survey.

\section{SIMULATION RESULTS}

The Mersim model produced a wide range of results. Due to the validation focus of this paper we concentrate on two results. The first concerns the simulation of PES to reduce the area under monoculture rubber plantations in Xishuangbanna in Yunnan, China. Chinese participants selected this decision making context as initial investigations revealed that rubber plantations exceeded governmental limits by $100 \%$ and were encroaching on national parks of high biodiversity value. Central and Province governments introduced a PES scheme to create an incentive for rubber farmer to reduce rubber plantings, to diversify their plantations by planting native trees and as a corollary, protect biodiversity. From a stakeholder perspective the principle PES design was perceived to be effective but the actual payments not sufficient to fully compensate income losses. Mersim simulations suggested that an increase of payments sufficient to fully compensated rubber farmers would increase the area under rubber. This counterintuitive result emerges from the fact that households in marginal areas perceive the payment as a form of subsidy and commence planting (diversified) rubber, accelerating the expansion of rubber into higher altitudes (Fig. 3).

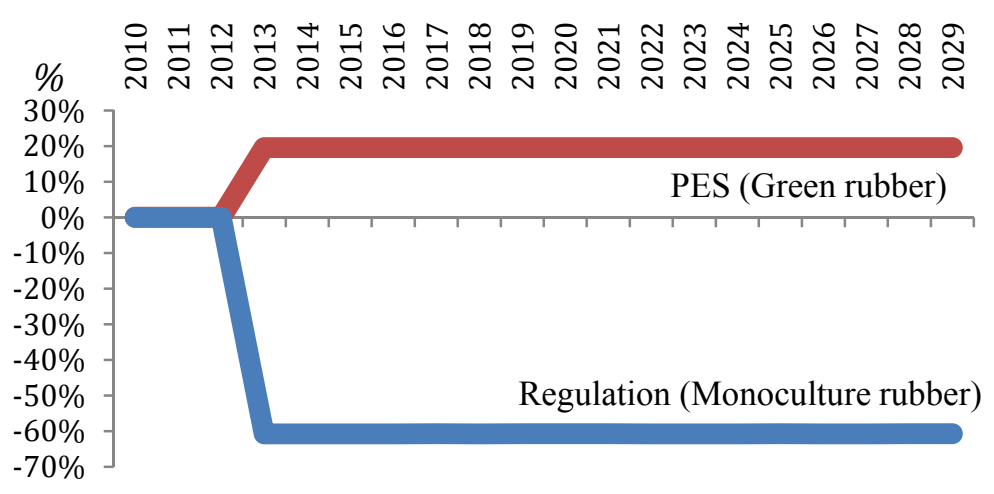

Fig. 3. Impacts of Government payments and regulation on area under rubber (change depicted in per cent) in Xishuangbanna, Yunnan 
The second set of results is focused on effects of irrigation on poverty. In Northeast Thailand and in Lao's Nam Ngum catchment large-scale investments in water-diversion and reservoirs are justified by increasing irrigation potential that will ultimately reduce poverty. Mersim simulations show that contrary to expectations, poverty levels remain largely unchanged as shown in Fig. 4. The benefits of improved irrigation potential accrue to land owners; the larger the land, the larger the additional benefit. However, the majority of poor households do not own land. Additional economic multiplier effects such as increased farm labour seem to be marginal in most districts simulated across Thailand and Lao PDR due to barter networks (non-monetary trade) and cheaper immigrants (Myanmar, Cambodia).
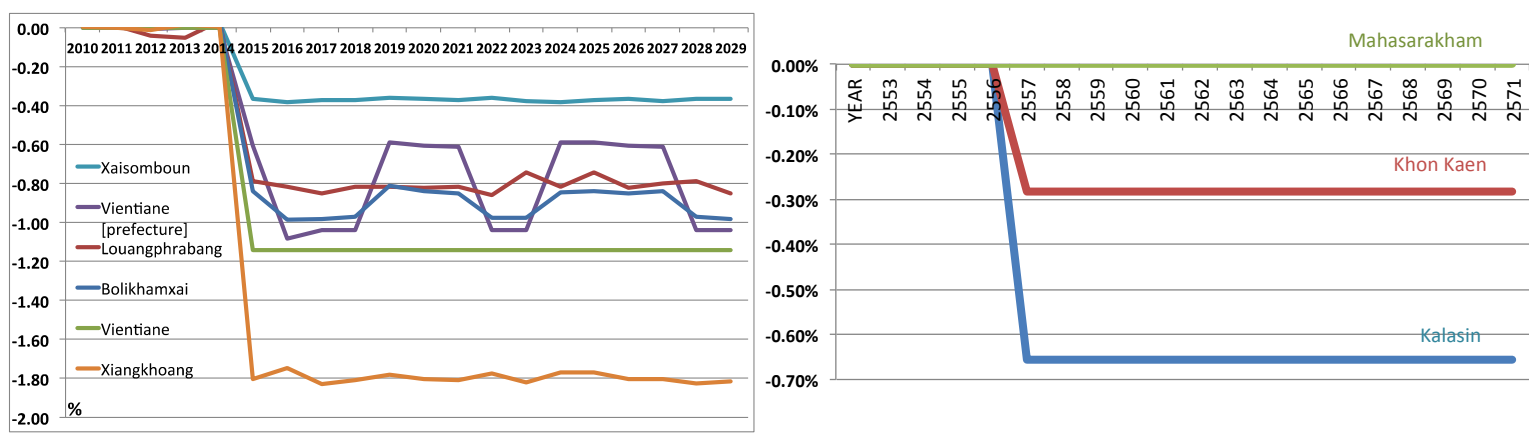

Fig. 4. Impacts of large-scale irrigation in the Nam Ngum catchment, Lao PDR, on poverty (left chart), and in Northeast Thailand (right chart); ordinate shows impacts on poverty in per cent and abscissa shows years.

\section{PATTERN-BASED VALIDATION}

Traditional modelling aims for the validation of numerical results to improve the robustness of predictive modelling capacity. In the case of the Mersim model, numerical validation was employed for evaluating the modelled performance of simulating the business-as-usual reference values. Primarily the poverty indicator was shown to experts for assessing the reliability of estimates of the numerical value of poverty and the spatial distribution. This is important as land use maps were in some parts highly aggregated, introducing the risk that the wrong livelihoods were placed in particular areas, such as urban or mountainous areas. In all three cases - Xishuangbanna, Nam Ngum and Isan - experts confirmed that Mersim simulated poverty levels and distributions represented a realistic facsimile of actual values and distributions.

The pattern-based validation, which aligns with the pattern-based modelling promoted by Grimm et al. (2005), was integrated into a larger learning approach and as part of a stakeholder workshop process. In preparation for this pattern-based validation, simulation results for the policy scenarios were plotted as graphs for each of the local study areas and as maps. The most relevant indicator for the participatory process was poverty in Lao PDR and Thailand and the area under rubber in Yunnan, China. Modelling results varied due to the number of stochastic parameters, therefore 200 model runs established a stable average and stable standard deviations. The analysis focused on identifying principle patterns that emerge independently from the numerical differences between individual runs. A suite of patterns emerged, ranging from simple causal patterns to spatial patterns. For the purpose of this paper we focus on causal patterns as described in the results section. For Thailand and Lao PDR one of the most debated patterns suggests there is no identifiable causal relationship between the extent of irrigation and poverty levels, at least for the majority of districts. For Xishuangbanna the key pattern was that the area under rubber initially expanded once PES schemes were activated.

These patterns were presented in stakeholder workshops in the context of stakeholder beliefs. During the three-year process one of the most influential beliefs expressed by participants in Lao PDR and Thailand was that irrigation was an effective poverty alleviation strategy. Similarly, Chinese stakeholders articulated repeatedly that the PES scheme they had implemented would be an effective strategy to curb the expansion of rubber. The learning methodology this participatory process implemented (Smajgl et al., in review; Smajgl and Ward, in review-b) sought to challenge existing beliefs, including the two beliefs outlined. The emerging patterns derived from the simulation model formed the analytical foundation to challenge existing beliefs. The stakeholders were first presented with their own beliefs, expressed and documented in previous workshops, which were discussed in plenary and break-out groups. Then, the simulation results were presented in the form of numerical results and the principal (causal) pattern. In all three local study processes, 
the results were initially rejected. However, once the simulation dynamics that explain the result were clarified, all stakeholders confirmed that the simulated results were consistent with their expectations. The explaining model mechanism for the counter-intuitive irrigation impact on poverty is the fact that land ownership is a prerequisite to deriving benefits from access to irrigation infrastructure. For the PES case stakeholders confirmed that the same counter-intuitive effect emerged with other government payments. The collective stakeholder response validated the key simulation results.

\section{DISCUSSION}

Highly complex decision making situations often demand complex methods. With increasing complexity in scientific methods a growing gap between science and policy needs to be bridged and the creation of confidence in the scientific process, methods and its results demand careful design. Validation is a critical step for all modelling-related activities, such as the model-supported learning process described in this paper. While numerical validation alone can be challenging in highly complex models (Moss, 2008; Moss and Edmonds, 2005; Oreskes et al., 1994; Smajgl et al., 2011b) it can be useful to determine model robustness with experts simulating business-as-usual or back-casting cases, which combine so-called 'Turing tests' and historical validity (David, 2013). Eliciting (logical, numerical or spatial) patterns is a key approach in validating simulation results emerging from the Mersim model. The validation and the learning process were interwoven and allowed for effectively challenging stakeholders assumptions on impacts of decision-making options while validating some of the most relevant (counter-intuitive) simulation results.

\section{REFERENCES}

Alcamo, J., 2001. Scenarios as tools for international assessments. European Environment Agency: Luxembourg.

Amblard, F., Bommel, P., Rouchier, J., 2007. Assessment and validation of multi-agent models, In: Phan, D., Amblard, F. (Eds.), Agent-based modelling and simulation in the social and human sciences. The Bardwell Press: Oxford, pp. 93-114.

Barreteau, O., LePage, C., D'Aquino, P., 2003. Role-playing games, models and negotiation processes. JASSS - The Journal of Artificial Societies and Social Simulation 6(2).

Bousquet, F., Barreteau, O., LePage, C., Mullon, C., Weber, J., 1999. An environmental modelling approach. The use of multi-agent simulations, In: Blasco, F., Weill, A. (Eds.), Advances in environmental and ecological modelling. Elsevier: Paris, pp. 113-122.

David, N., 2013. Validating Simulations, In: Edmonds, B., Meyer, R. (Eds.), Simulating Social Complexity. Springer: Berlin, Heidelberg, pp. 135-171.

Foran, T., Ward, J., Kemp-Benedict, E., Smajgl, A., in print. Developing detailed foresight narratives: a participatory technique from the Mekong region. Ecology and Society.

Gilbert, N., 2008. Agent-based models. SAGE Publications, Los Angeles.

Grimm, V., Berger, U., Bastiansen, F., Eliassen, S., Ginot, V., Giske, J., Goss-Custard, J., Grand, T., Heinz, S.K., Huse, G., Huth, A., Jepsen, J.U., Jørgensen, C., Mooij, W.M., Müller, B., Pe'er, G., Piou, C., Railsback, S.F., Robbins, A.M., Robbins, M.M., Rossmanith, E., Rüger, N., Strand, E., Souissi, S., Stillman, R.A., Vabø, R., Visser, U., DeAngelis, D.L., 2006. A standard protocol for describing individual-based and agentbased models. Ecological Modelling 198(1-2) 115-126.

Grimm, V., Berger, U., DeAngelis, D.L., Polhill, J.G., Giske, J., Railsback, S.F., 2010. The ODD protocol: A review and first update. Ecological Modelling 221(23) 2760-2768.

Grimm, V., Revilla, E., Berger, U., Jeltsch, F., Mooij, W.M., Railsback, S.F., Thulke, H.-H., Weiner, J., Wiegand, T., DeAngelis, D.L., 2005. Pattern-Oriented Modeling of Agent-Based Complex Systems: Lessons from Ecology. Science 310(5750) 987-991.

Matthews, R., Gilbert, N., Roach, A., Polhill, J., Gotts, N., 2007. Agent-based land-use models: a review of applications. Landscape Ecology 22(10) 1447-1459.

Moss, S., 2008. Alternative Approaches to the Empirical Validation of Agent-Based Models. Journal of Artificial Societies and Social Simulation 11((1) 5).

Moss, S., Edmonds, B., 2005. Sociology and Simulation: Statistical and Qualitative Cross-Validation. American Journal of Sociology 110(4) 1095-1131.

Oreskes, N., Shrader-Frechette, K., Belitz, K., 1994. Verification, Validation, and Confirmation of Numerical Models in the Earth Science. Science 263 641-646.

Sawyer, R.K., 2005. Social Emergence: Societies as Complex Systems. Cambridge University Press, New York.

Smajgl, A., 2010. Challenging beliefs through multi-level participatory modelling in Indonesia. Environmental Modelling and Software 25(11) 1470-1476. 
Smajgl, A., Bohensky, E., 2013. Behaviour And Space In Agent-Based Modelling: Poverty Patterns In East Kalimantan, Indonesia. Environmental Modelling and Software 45 8-14.

Smajgl, A., Brown, D.G., Valbuena, D., Huigen, M.G.A., 2011a. Empirical characterisation of agent behaviours in socio-ecological systems. Environmental Modelling \& Software 26(7) 837-844.

Smajgl, A., Egan, S., Ward, J., Kroon, F., 2013. The Mekong region simulation (Mersim) model - Design Document CSIRO Climate Adaptation Flagship: Townsville.

Smajgl, A., Foran, T., Dore, J., Ward, J., Larson, S., in review. Visions, beliefs and transformation: Methods for understanding cross-scale and trans-boundary dynamics in the wider Mekong region. Global Environmental Change.

Smajgl, A., Heckbert, S., Ward, J., Straton, A., 2009a. Simulating impacts of water trading in an institutional perspective. Environmental Modelling \& Software 24(2) 191-201.

Smajgl, A., House, A., Butler, J., 2011b. Implications of ecological data constraints for integrated policy and livelihoods modelling: an example from East Kalimantan, Indonesia Ecological Modelling 222 888-896.

Smajgl, A., Izquierdo, L., Huigen, M., 2008. Modelling endogenous rule changes in an institutional context: The ADICO sequence. Advances in complex systems 2(11) 199-215.

Smajgl, A., Morris, S., Heckbert, S., 2009b. Water policy impact assessment - combining modelling techniques in the Great Barrier Reef region. Water policy 11 191-202.

Smajgl, A., Ward, J., 2013. The Water-Food-Energy Nexus in the Mekong Region. Springer: New York.

Smajgl, A., Ward, J., in review-a. A design protocol for research impact evaluation: Development investments of the Mekong region. Research Evaluation.

Smajgl, A., Ward, J., in review-b. A framework for bridging Science and Decision making. Futures.

Troitzsch, K.G., 2013. Historical Introduction, In: Edmonds, B., Meyer, R. (Eds.), Simulating Social Complexity. Springer: Berlin, Heidelberg, pp. 13-21.

Windrum, P., Fagiolo, G., Moneta, A., 2007. Empirical Validation of Agent-Based Models: Alternatives and Prospects. Journal of Artificial Societies and Social Simulation 10((2) 8).

Young, H.P., 2006. Chapter 22 Social Dynamics: TheorY AND Applications, In: Tesfatsion, L., Judd, K.L. (Eds.), Handbook of Computational Economics. Elsevier, pp. 1081-1108. 